H I G H L I G H T S

APOPTOSIS

\section{Pour or pore?}

Although nobody doubts that the release of cytochrome $c$ from mitochondria is a crucial step in cell death, quite how this happens is a source of controversy. Some say that the proapoptotic molecule Bax punches holes directly in the outer mitochondrial membrane (OMM) through which cytochrome $c$ pours out. But others believe that Bax acts more indirectly, cooperating with components of the permeability transition pore (PTP). This could cause the swelling and rupture of mitochondria (and the subsequent release of cytochrome $c$ ), or it might allow the pore to open widely enough to allow cytochrome $c$ to pass through.

A report by Donald Newmeyer and co-workers in Cell now provides evidence for the first theory, showing that activated Bax can mediate the formation of supramolecular openings in the OMM. The authors did this by developing cell-free systems that allowed them to reproduce the behaviour of whole mitochondria.

Newmeyer and co-workers first isolated Xenopus OMMs and resealed them to form vesicles. Although these vesicles probably contained most of the proteins found in the OMM (including components of the PTP), they were devoid of inner-mitochondrial-membrane (IMM) and mitochondrialmatrix proteins. The authors then showed that fluoroscein dextrans ranging in size from $10-2,000 \mathrm{kDa}-$ could be released from these vesicles on treatment with either oligomerized (and therefore active) Bax (OG-Bax), or a cleaved, active form of another pro-apoptotic molecule Bid (N/CBid). Conversely, dextran release could be blocked by treatment with the antiapoptotic protein $\mathrm{Bcl}-\mathrm{X}_{\mathrm{L}}$.

There was no gross disruption of the lipid bilayer, which indicates that supramolecular openings might somehow have been created in the membrane. But could N/C-Bid and OGBax do this in the absence of other proteins? To test this the authors generated two types of protein-free liposome - one from extracted mitochondrial lipids ('M liposome') and one from

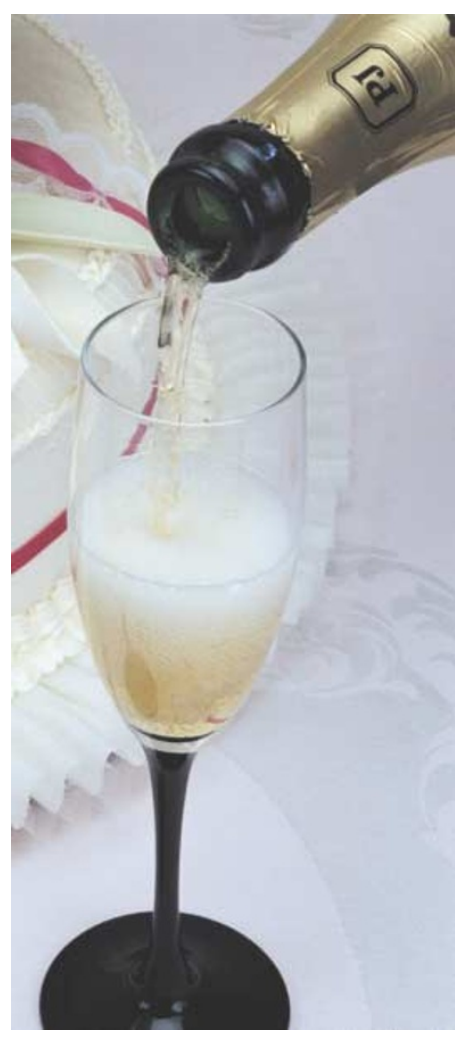

microsomal lipids ('ER liposome'). Dextran release triggered by OG-Bax was much more efficient from M liposomes than from ER liposomes. N/C-Bid alone did not trigger dextran release from either type of liposome; however, when N/C-Bid and monomeric Bax were added together there was efficient release from the $\mathrm{M}$ (but not the ER) liposomes. N/C-Bid can therefore activate monomeric Bax to produce the membrane openings.

The main difference between the two types of liposome is the additional presence of cardiolipin in the M liposomes. So the authors suggest that cardiolipin is important for both the membrane-targeting ability of Bid and the permeabilization function of Bax. Interestingly, however, the IMM which is not permeabilized by Bax during apoptosis - is also rich in cardiolipin. One explanation, proposed by Newmeyer and co-workers, is that because the cardiolipin might be in the inner leaflet of the IMM, it would not be accessible to Bax. Further studies using the new cell-free systems should be able to address this question. Alison Mitchell

(4) References and links ORIGINAL RESEARCH PAPER Kuwana, T. etal. Bid, Bax, and lipids cooperate to form supramolecular openings in the outer mitochondrial membrane. Cell 111, 331-342 (2002)

\section{STRUCTURE WATCH}

\section{A solid technique}

When we think of techniques for determining three-dimensional (3D) protein structure, X-ray crystallography and solution NMR probably first come to mind. But, what do we do when proteins are inaccessible to solution NMR or do not readily crystallize? Could solid-state magic-angle-spinning (MAS) NMR be a solution? To determine a 3D NMR structure, you need a large number of distance restraints, but for solid-state NMR — which could be applied to the tricky situations above - there has been no easy way to collect these restraints. Oschkinat and co-workers therefore devised a technique that allowed them to determine the structure of the $\alpha$-spectrin Src-homology 3 (SH3) domain using only solidstate MAS NMR. Restraints between carbon atoms are essential for defining a protein's 3D structure, but so-called dipolar truncation effects make it difficult to measure these restraints in fully labelled ${ }^{13} \mathrm{C}$ samples. The authors therefore made three differently labelled forms of the $\alpha$-spectrin SH3 domain - one fully labelled sample and two biosynthetically site-directed ${ }^{13} \mathrm{C}$-enriched samples. Altering the labelling pattern in the latter two samples reduced the problem of dipolar truncation effects and allowed the observation of long-range interactions in the resulting NMR spectra.

The 3D structure of the $\alpha$-spectrin SH3 domain determined by solid-state MAS NMR is remarkably similar to the X-ray structure, and this work "...paves the way for the structure determination of amyloids, small membrane proteins or receptor-ligand complexes...".

REFERENCE Castellani, F. et al. Structure of a protein determined by solid-state magicangle-spinning NMR spectroscopy. Nature 420, 98-102 (2002)

\section{Strong ties}

Pseudomonas aeruginosa can infect most human tissues when the immune system is compromised, and this opportunistic pathogen is particularly dangerous to cystic fibrosis patients. In these patients, cell-surface glycoconjugates are increasingly fucosylated, and this makes them targets for $P$. aeruginosa, because this bacterium makes large amounts of a lectin called PA-IIL that binds L-fucose with a high affinity. In Nature Structural Biology, Imberty and colleagues now increase our understanding of PA-IIL by reporting its highresolution crystal structure in complex with fucose. In addition, they used binding and modelling studies to show that antigens of the Lewis a series might be the preferred ligands of PA-IIL.

The crystal structure of PA-IIL is a tetramer, and the four independent monomers each consist of a nine-stranded antiparallel $\beta$-sheet. Each monomer binds two $\mathrm{Ca}^{2+}$ ions and one fucose ligand, and the authors found that "...fucose locks onto both calcium ions, a binding mode that is unique among protein-carbohydrate interactions." This unique interaction explains the high affinity of PA-IIL for fucose - for most C-type animal lectins, which also bind two $\mathrm{Ca}^{2+}$ ions, only one ion is involved in sugar binding.

The strong ties between PA-IIL and fucose indicate that it could be a target for oligosaccharide-based therapeutics, and the structural detail provided by Imberty and co-workers should help in the design of antibacterial-adhesion prophylactics.

REFERENCE Mitchell, E. et al. Structural basis for oligosaccharide-mediated adhesion of Pseudomonas aeruginosa in the lungs of cystic fibrosis patients. Nature Struct. Biol. 9 , 918-921 (2002) 